Article

\title{
Pricing Basket Weather Derivatives on Rainfall and Temperature Processes
}

\author{
Nelson Christopher Dzupire ${ }^{1, * \mathbb{D}}$, Philip Ngare ${ }^{1,2}$ and Leo Odongo ${ }^{1,3}$ \\ 1 Mathematics Department, Pan African University Institute of Basic Sciences, Technology, and Innovation, Juja, \\ Nairobi 00200, Kenya \\ 2 Department Actuarial Science, University of Nairobi, Nairobi 00100, Kenya \\ 3 Department of Statistics and Actuarial Science, Kenyatta University, Nairobi 00100, Kenya \\ * Correspondence: ndzupire@cc.ac.mw
}

Received: 7 March 2019; Accepted: 10 June 2019; Published: 27 June 2019

check for updates

\begin{abstract}
This paper follows an incomplete market pricing approach to analyze the evaluation of weather derivatives and the viability of a weather derivatives market in terms of hedging. A utility indifference method is developed for the specification of indifference prices for the seller and buyer of a basket of weather derivatives written on rainfall and temperature. The agent's risk preference is described by an exponential utility function and the prices are derived by dynamic programming principles and corresponding Hamilton Jacobi-Bellman equations from the stochastic optimal control problems. It is found the indifference measure is equal to the physical measure as there is no correlation between the capital market and weather. The fair price of the derivative should be greater than the seller's indifference price and less than the buyer's indifference price for market viability and no arbitrage opportunities.
\end{abstract}

Keywords: incomplete market; basket option; indifference price; utility function; dynamic programming principle; Hamilton Jacobi-Bellman equations

\section{Introduction}

In the context of climate change, rainfall and temperature are the major determinants of uncertainty affecting crop yield. Crop yields are strongly susceptible to extreme conditions such as drought, flood and heat waves Zscheischler et al. (2017). Temperature and rainfall processes are the key driving factors of crop yield. Typically, temperature determines the duration of the growing season, whereas rainfall has impact on plant production (leaf area and the photosynthetic efficiency) Medori et al. (2012).

Ray et al. (2015) estimated that globally variation in temperature and precipitation accounts for about a third of the crop yield variability. Besides agriculture, weather risks have a major influence in many economic sectors as several companies are weather dependent. However, the interdependence between these two variables proves it is difficult to accurately analyze and simulate their joint behavior. The impact of rainfall on moisture in turn controls the partition between the sensible and latent heat fluxes Cong and Brady (2012).

This draws attention to contingency planning such as establishing contingency funds, increasing forex reserves for external shocks and piloting the use of weather insurance Syroka and Nucifora (2010). Similarly, weather derivatives sold to small scale farmers may act as risk management tool at household level against poor yields.

To overcome the loss of crop yield due to weather factors, a farmer can explore the possibility of hedging the risks due to weather by buying weather derivatives that guarantee a payoff once there is 
erratic rainfall or temperature. For this to be effective, a better understanding of weather dynamics, especially temperature and rainfall processes, and possible hedging mechanisms would therefore offer a new way of transferring financial risks in the uncertainties of weather and climate to financial markets where risk management strategies exist.

A weather derivative is a financial instrument used by companies or individuals to hedge against the risk of weather related losses Alexandridis and Zapranis (2012). Weather derivatives have an underlying index that measures a particular aspect of weather such as total accumulated rainfall, number of cooling degree days, etc. over a specific period of time. They cover low risk, high probability events unlike weather insurance that deals with high risk, low probability events. In agriculture, a farmer may buy a weather derivative to hedge against poor yields caused by too much or too little rainfall, sudden temperature changes, or destructive winds. In terms of weather risk management, weather derivatives serve a significant role considering the global exposure of large businesses to increasingly uncertain global weather conditions in sectors such as energy, agriculture, tourism, insurance and retail. This is because weather derivatives are economical, flexible and require no proof of loss Brockett et al. (2009).

Several studies have been carried on weather derivative evaluation and pricing procedures, e.g., on rainfall Cao et al. (2004); Carmona and Diko (2005); López Cabrera et al. (2013); Leobacher and Ngare (2011); Odening et al. (2007); Xu et al. (2007) (and the references therein) and temperature Benth and Šaltytė-Benth (2005); Benth and Šaltyte Benth (2011); Brody et al. (2002); Cui (2014); Wang et al. (2015). Typically, these studies only consider a single weather element-rainfall, temperature or wind-in the pricing and evaluation.

However, weather derivatives present a new market, thus analysis and examination of market viability provide important insights for investors. Hence, in this paper, we explore the possibility of basket weather derivatives whose underlying indexes are rainfall and temperature, both of which are not tradable. A basket option is a financial derivative where the underlying asset is a group of commodities, securities or currencies. The simultaneous role of rainfall and temperature in agriculture cannot be underrated, as it is common knowledge that crop yield greatly depends on them. Turvey, Calum G (2001) and Ray et al. (2015) independently concluded that up to $30 \%$ of crop yield variability can be attributed to heat and rainfall events, therefore temperature and rainfall remain the driving force in agriculture.

There are no known basket weather derivatives and their application in agriculture or any other weather related industries currently and therefore this paper aims to fill that gap. A better understanding of the dynamics driving both the rainfall and temperature processes and possible hedging approaches provide a new way to transfer financial risks due to weather to financial markets where risk management strategies are available. This basket weather derivative enables the farmer to hedge risk due to rainfall and temperature variation at the same time in one transaction and to do it more cheaply rather than hedging rainfall and temperature independently. Such a derivative is more applicable as the likelihood of both temperature and rainfall variation to affect yield is higher.

The paper is structured into three parts. In Section 2, we look at pricing approaches in an incomplete market and the challenges of pricing weather derivatives in an incomplete market where no arbitrage theory prices cannot be used. In Section 3, we price the weather derivatives using the utility indifference pricing method. We derive the seller's and buyer's indifference price using dynamic programming principles where three stochastic optimal control problems are solved with the exponential utility function as the investor's risk preference. The conditions for market viability and no arbitrage opportunities are also stated. Finally, we conclude the study in Section 5. 


\section{Pricing in an Incomplete Market}

A market is said to be complete if all contingent claims can be replicated by a self financing portfolio. By replication, we mean a process where a portfolio in stocks and bonds recreates the terminal pay-off of the option, thus removing all risks and uncertainty. In a complete market, the price of a contingent claim $F$ which depends on a stochastic variable $I$ and expires at time $T$ is given as

$$
F=e^{-r T} \mathbb{E}_{\mathbb{Q}}\left[W_{T}(I)\right],
$$

where $W_{T}(I)$ is the pay-off of the derivative, $\mathbb{E}$ is the expectation conditioned on the present information, and $\mathbb{Q}$ are the risk neutral probabilities Xu et al. (2007). Using the Radon-Nikodym derivative of $\mathbb{Q}$ with respect to the physical probabilities $\mathbb{P}$, Equation (1) can be expressed as

$$
F=e^{-r T} \mathbb{E}_{\mathbb{P}}\left(\frac{d \mathbb{Q}}{d \mathbb{P}} W_{T}(I)\right)
$$

This change makes the stochastic process of $I$ into a martingale. If the stock follows a geometrical Brownian motion, change of measure can be achieved by reducing the drift to the risk free interest rate.

However, weather indexes are not tradable, therefore the market is incomplete as the derivatives cannot be replicated by a self financing portfolio. This makes it impractical to apply no arbitrage pricing models to weather derivatives Alexandridis and Zapranis (2012); Benth and Benth (2007) as we cannot construct a risk free portfolio consisting of weather index and the derivative. In addition, the no arbitrage condition does not result in a unique price as many martingale measures exists, thus only bounds for contingent claims can be obtained Xu et al. (2007). Formally, we have the range

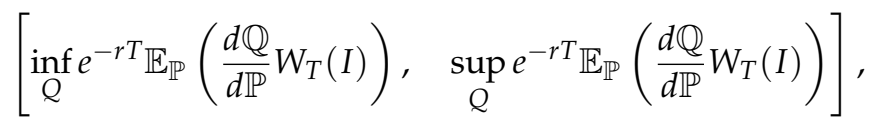

where $Q$ denotes the set of all equivalent martingale measures where the interval in Equation (3) is very large and hence not useful Eberlein and Jacod (1997). In an incomplete market, the investor aspires to maximize the expected utility of final wealth and reduce the risks due to the uncertain pay-off through dynamic trading Carmona (2008). Hence, the goal is to find a trading strategy that minimizes the risk quantified by a risk measure while maximizing the expected utility of terminal wealth under the physical measure.

Several approaches are used to price contingent claims in an incomplete market and there is no consensus about the best approach since all existing approaches have their particular shortfalls ranging from no theoretical basis to some requiring several assumptions to make them tractable. The approaches include utility indifference pricing, super-replication, quadratic approaches, quantile hedging, shortfall minimization, and marginal utility approach (Brockett et al. (2009); Henderson and Hobson (2004); $\mathrm{Xu}$ et al. (2007) and the references therein).

In this study, we price the basket weather derivatives using utility indifference approach to derive the buyer's and sellers' price. As observed by Xu et al. (2007), the indifference approach starts with an appealing idea that the amount of money at which a potential buyer (seller) of a claim is indifferent in terms of expected utility between buying (selling) and not buying (selling) constitutes an upper (lower) limit for the contract price. The utility indifference price approach is used because it incorporates risk aversion in the model, results in a nonlinear price model, and reduces to the complete price, which is a necessary feature of any good price mechanism Henderson and Hobson (2004). 


\section{Indifference Pricing Approach}

In this study, we want to price a contingent claim written on rainfall and temperature, both of which are weather indexes that are not traded but have huge influence on agriculture products.

Historical daily rainfall and daily average temperature data are used to predict the future behavior of the weather elements. We assume a static option in that the portfolio dynamics cannot be changed once the buyer and the seller agree on the prices. The basket option is held for the whole growing season entire contract duration regardless of the current weather data being experienced. In that way, the model is time consistent such that, if at some point in the future one option costs more than the other option in every world state, the same should be true today.

The intensity of rainfall is modeled as a Gamma distributed with the probability density function as

$$
f(R)=\frac{\alpha^{P} R^{P-1} e^{-\alpha R}}{\Gamma(P)} \quad R>0
$$

which is a strong stationary process with fixed mean and variance. This is equivalent to

$$
f(R)=\frac{R^{P-1}}{\Gamma(P) \gamma^{P}} e^{-\frac{R}{\gamma}}, \quad \gamma=\frac{1}{\alpha}
$$

such that $P$ is shape parameter and $\gamma$ is a scale parameter.

To model the rainfall intensity process as a mean reverting process, the probability density function is transformed to a stationary Gamma probability density function by introducing the location parameter $\mu$ and a scale parameter $\lambda$, which represents the speed of decrease towards equilibrium in the stochastic differential equation. Hence, the probability density function becomes

$$
\begin{aligned}
f(R) & =\frac{\lambda^{P}(R-\mu)^{P-1}}{\Gamma(P) \gamma^{P}} e^{-\lambda\left(\frac{R-\mu}{\gamma}\right)} \\
\gamma & >0, \quad \lambda>0, \quad P>0, \quad \mu<R<\infty,
\end{aligned}
$$

Based on the approach by Hertzler (2003), given $f(R)$ we have to find the functions $g(R)$ and $h(R)$ such that

$$
d R=\varphi(R) d t+v(R) d W
$$

where $d W$ is the Wiener process and satisfies the Kolmogorov forward equation:

$$
\frac{\partial[\varphi f]}{\partial R}-\frac{1}{2} \frac{\partial^{2}}{\partial R^{2}}\left[v^{2} f\right]=0
$$

Integrating Equation (4), we have

$$
\varphi f-\frac{1}{2} \frac{\partial\left[v^{2} f\right]}{\partial R}=0
$$

The choice of $f$ and $h$ is heuristic and hence we verify whether the chosen functions satisfy the Kolmogorov forward equation. Letting $v=\sqrt{2 \gamma}$ and $\varphi=-\lambda+(P-1)(R-\mu)^{-1} \gamma$, we have 


$$
\begin{aligned}
\varphi f & =-\lambda+(P-1)(R-\mu)^{-1} \gamma\left[\frac{\lambda^{P}(R-\mu)^{P-1}}{\Gamma(P) \gamma^{P}} e^{-\lambda\left(\frac{R-\mu}{\gamma}\right)}\right] \\
\frac{\partial\left[v^{2} f\right]}{\partial R} & =\frac{2 \gamma \lambda^{P}}{\Gamma(P) \gamma^{P}}\left[(P-1)(R-\mu)^{P-2} e^{-\lambda\left(\frac{R-\mu}{\gamma}\right)}-\frac{\lambda}{\gamma}\left((R-\mu)^{P-1} e^{-\lambda\left(\frac{R-\mu}{\gamma}\right)}\right],\right.
\end{aligned}
$$

thus we clearly have $\varphi f-\frac{1}{2} \frac{\partial\left[v^{2} f\right]}{\partial R}=0$.

Therefore, the stochastic differential equation driving the dynamics of rainfall intensity is

$$
\begin{aligned}
d R_{t} & =-\lambda+(P-1)(R-\mu)^{-1} \gamma d t+\sqrt{2 \gamma} d W_{t}^{1} \\
& =\varphi(R) d t+v(R) d W_{t}^{1} \quad \text { where } \\
R_{0} & =r \in \mathbb{R}
\end{aligned}
$$

where $R$ is the daily rainfall intensity.

For the temperature process, the statistical analysis involves removing long-term trends, global warming impact and the seasonal mean, which is cyclic. The remaining residual is an autoregressive model whose order depends on location; however, since the marginal distribution tail is heavier than normal, we represent it more generally without constant and deterministic volatility as in Carmona (2008), thus the dynamics are as follows:

$$
\begin{aligned}
d Y_{t} & =-k\left[Y_{t}-s_{t}\right] d t+\vartheta(t) d W_{t}^{2} \\
& =\eta(Y, t) d t+\vartheta(t) d W_{t}^{2} \\
Y_{0} & =y \in \mathbb{R},
\end{aligned}
$$

where $Y$ is the average daily temperature, $s_{t}$ is the cyclic season mean and $\vartheta(t)$ is the volatility function.

Since there is interdependence between the temperature and rainfall processes, we have a correlation between $W_{t}^{1}$ and $W_{t}^{2}$ with a correlation parameter $\rho \in[-1,1]$. The market model consists of a risky asset $S$ that is traded and presence of temperature and rainfall indexes on which a European basket option is written. The tradable asset has a price dynamics as follows:

$$
\begin{aligned}
d S_{t} & =\mu S_{t} d t+\sigma S_{t} d W_{t}^{3} \\
S_{0} & =s \in \mathbb{R} .
\end{aligned}
$$

The processes $W_{t}^{1}, W_{t}^{2}, W_{t}^{3}$ are Wiener processes defined on a filtered probability space $\left[\Omega, \Im,(\Im)_{0 \leq t \leq T}, \mathbb{P}\right]$, where $(\Im)$ is $\sigma$-algebra generated by $\left\{W_{u}^{1}, W_{u}^{2}, W_{u}^{3}: 0 \leq u \leq t\right\}$.

The relevant conditions for the existence and uniqueness of the solution to Equations (5) and (6) are summarized in Lemma 1.

Lemma 1. Bjork (2009) Suppose there exists a constant $K$ such that the following conditions are satisfied $\forall x, y, t$

$$
\begin{array}{r}
\|\mu(t, x)-\mu(t, y)\| \leq K\|x-y\| \\
\|\sigma(t, x)-\sigma(t, y)\| \leq K\|x-y\| \\
\|\mu(t, x)\|-\|\sigma(t, x)\| \leq K(1+\|x\|),
\end{array}
$$

then there exists a unique solution to Equations (5) and (6) that is $\Im_{t}^{W_{t}^{3}}$ adapted and a Markovian process. 
It is also assumed that there is a riskless bond that matures at $T$ available for trading under a constant rate $r, 0 \leq r<\mu$, so that the bond price is

$$
d B_{t}=r B d t
$$

Weather derivatives are constructed to hedge risks due to weather factors such as rainfall, temperature, wind, etc. for a specific period of time. In this paper, we design a European basket option that is written in terms of cumulative rainfall and cooling degree days simultaneously. We assume that, for a growing season, the maize requires a specific amount of rainfall cumulatively and also that it grows well on specific temperature during the season. Hence, the option takes into account the difference between the recommended amount of rainfall and the cumulative rainfall of the season as well as accounts for days on which temperature is way above the recommended one. We assume that hedgers of this weather derivative hold it for the whole growing season up to maturity date.

The investor starts with initial wealth of $x$ at time $t$ and re-balances his portfolio by dynamically deciding the investments allocation $\pi_{s}^{b}$ and $\pi_{s}$ in the bond and risky asset, respectively, where we do not allow intermediate consumption or infusion of funds. Hence, the current wealth is defined by

$$
X_{s}=\pi_{s}^{b}+\pi_{s}, \quad t \leq s \leq T
$$

Proposition 1. The budget equation for the investor is given by

$$
\begin{aligned}
d X_{s} & =r X_{s} d s+(\mu-r) \pi_{s} d s+\sigma \pi_{s} d W_{s}^{3}, \quad t \leq s \leq T \\
X_{t} & =x \in \mathbb{R} .
\end{aligned}
$$

Proof. The wealth dynamics is $d X_{s}=\phi_{s} d S_{s}+\psi_{s} d B_{s}$, where $\phi_{t}$ and $\psi_{t}$ are the number of shares of risky asset and the bond, respectively, held by the investor. For $\pi_{s}$ amount of money in risky asset, we have

$$
\pi_{s}=\phi_{s} S_{s}, \quad \psi_{s}=X_{s}-\pi_{s}=X_{s}-\phi_{s} S_{s}
$$

Then,

$$
\begin{aligned}
d X_{s} & =\phi_{s} d S_{s}+\psi_{s} d B_{s} \\
& =\frac{\pi_{s}}{S_{s}} d S_{s}+\frac{X_{s}-\pi_{s}}{B_{s}} d B_{s} \\
& =\frac{\pi_{s}}{S_{s}}\left[\mu S_{s} d s+\sigma S_{s} d W_{s}^{3}\right]+\frac{X_{s}-\pi_{s}}{B_{s}}\left[r B_{s} d s\right] \\
& =r X_{s} d s+(\mu-r) \pi_{s} d s+\sigma \pi_{s} d W_{s}^{3}, \quad t \leq s \leq T,
\end{aligned}
$$

where $X_{t}=x$ is the initial wealth.

$\pi_{s}$ is deemed admissible if there exists a unique positive wealth process solving Equation (7) such that

$$
\int_{t}^{T} \pi_{s}^{2}\left(X_{s}^{\pi}\right)^{2} d s<\infty
$$

We denote the set of all admissible controls as $\Pi$. We model the agent's risk preference using exponential utility function defined as

$$
U(x)=-e^{-\gamma x}, \quad \gamma>0, x \in \mathbb{R},
$$


where $\gamma$ is the risk aversion of the agent representing his or her attitude towards the risk that cannot be eliminated. Any chosen utility function should be increasing to reflect the investor's preference of more wealth and concave as the investor is risk averse. Other utility functions such as power, mean-variance or $\log$ functions may be applied as well to describe the investor's risk preference. The exponential utility function is chosen based on that risk neutral measure implied by the indifference pricing equals the minimum entropy measure $\mathrm{Xu}$ et al. (2007).

The main objective is to price the weather derivative $W_{T}(R, Y)$ as the agent tries to hedge away the risk associated with the derivative with a portfolio invested in the tradable asset $S$. This entails an optimization problem where we find an admissible strategy $\pi^{*}$ (optimal) such that the utility derived from the wealth is maximized at the final time $T$ with and without the weather derivative respectively.

To make the most significant parameters of the model in the budget equation more transparent, we assume $r=0$ so that dynamics of the bond are removed from the model. The valuation of the weather derivative is based on the comparison of maximal expected utility pay-off corresponding to the investment opportunities with and without the weather derivative. This entails formulating a stochastic optimal control problem, deriving and linearizing a Hamilton-Jacobi-Bellman (HJB) equation and stating the stochastic representation of the solution. Similar approaches were done by Benth and Karlsen (2005); (Musiela and Zariphopoulou 2001, 2003, 2004), however, unlike all these researchers, our work here considers presence of two non-tradable weather indexes on which the European option is written. The alternative method to solve such stochastic problems is to use martingale approach; however, it was observed by Musiela and Zariphopoulou (2001) that the approach produces limited results in an incomplete market setting, hence is not reliable to use.

The indifference price of the contingent claim $W_{T}(R, Y)$ is constructed from three stochastic optimal control problems as follows:

The first problem is where the agent maximizes the expected utility wealth without the contingent claim $W_{T}(R, T)$, commonly known as the classical Merton model of investment with the value function defined as

$$
V(x, t)=\sup _{\pi \in \Pi} \mathbf{E}\left[-e^{-\gamma X_{T}} \mid X_{t}=x\right] .
$$

Taking into account the contingent claim $W_{T}(R, Y)$, we have the following value functions for the seller and the buyer, respectively,

$$
\begin{aligned}
V^{s}(x, r, y, t) & =\sup _{\pi \in \Pi} \mathbf{E}\left[-e^{-\gamma\left[X_{T}-W_{T}(R, Y)\right]} \mid X_{t}=x, R_{t}=r, Y_{t}=y\right], \\
V^{b}(x, r, y, t) & =\sup _{\pi \in \Pi} \mathbf{E}\left[-e^{-\gamma\left[X_{T}+W_{T}(R, Y)\right]} \mid X_{t}=x, R_{t}=r, Y_{t}=y\right] .
\end{aligned}
$$

Definition 1. Musiela and Zariphopoulou (2004) The indifference price of the seller of the weather derivative is defined as a function $p^{s}(x, r, y, t)$ such that the investor is indifferent towards the following two cases: optimizing the expected utility without employing the derivative, and optimizing it taking into account on the one hand the liability $W_{T}(R, Y)$ at expiration $T$ and on the other hand the compensation $p^{s}(x, r, y, t)$ at inscription $t$. Mathematically,

$$
V(x, t)=V^{s}\left(x+p^{s}(x, r, y, t), r, y, t\right),
$$

and similarly the buyer's indifference price $p^{b}(x, r, y, t)$ satisfies

$$
V(x, t)=V^{b}\left(x-p^{b}(x, r, y, t), r, y, t\right) .
$$


Definition 2. A process $\pi$ in the form $\pi_{s}=a(s, X(s))$ for some measurable function

$$
a:[0, T] \times R^{n} \rightarrow R^{n}
$$

is called Markovian.

Restricting the optimization of the value functions in Equations (8)-(10) to Markovian admissible controls leads to the derivation of the HJB equation by dynamic programming principle Fleming and Soner (2006).

The corresponding HJB equation for the classical Merton problem value function $V$ is as follows

$$
\begin{aligned}
V_{t}+\max _{\pi}\left(\mu \pi V_{x}+\frac{1}{2} \sigma^{2} \pi^{2} V_{x x}\right) & =0 \\
V(x, T) & =e^{-\gamma x} .
\end{aligned}
$$

Differentiating $\mu \pi V_{x}+\frac{1}{2} \sigma^{2} \pi^{2} V_{x x}$ with respect to $\pi$, the optimal value $\pi^{*}$ is

$$
\begin{aligned}
\pi \sigma V_{x x}+\mu V_{x} & =0 \\
\pi^{*} & =-\frac{\mu}{\sigma} \frac{V_{x}}{V_{x x}} .
\end{aligned}
$$

Substituting into the HJB equation, we have

$$
\begin{aligned}
V_{t}-\frac{1}{2} \frac{\mu^{2}}{\sigma^{2}} \frac{V_{x}^{2}}{V_{x x}} & =0 \\
V(x, T) & =e^{-\gamma x} .
\end{aligned}
$$

By separation of variables approach, we suggest a candidate solution to the problem as $V(x, t)=-e^{-\gamma x} A(t)$ so that

$$
-e^{-\gamma x} A_{t}-\frac{1}{2} \frac{\mu^{2}}{\sigma^{2}}\left(\frac{\gamma^{2} e^{-2 \gamma x} A_{t}^{2}}{-\gamma^{2} e^{-\gamma x}}\right)=0
$$

Evaluating to

$$
\begin{aligned}
A_{t}-\frac{1}{2} \frac{\mu^{2}}{\sigma^{2}} A(t) & =0 \\
A(T) & =1 .
\end{aligned}
$$

Hence, this reduces to

$$
\begin{aligned}
\ln A(T)-\ln A(t) & =\frac{1}{2} \frac{\mu^{2}}{\sigma^{2}}(T-t) \\
\ln A(t) & =-\frac{1}{2} \frac{\mu^{2}}{\sigma^{2}}(T-t) \\
A(t) & =e^{-\frac{1}{2} \frac{\mu^{2}}{\sigma^{2}}(T-t)} .
\end{aligned}
$$


Proposition 2. Without weather hedging, the optimal value function and control are, respectively,

$$
\begin{aligned}
V(x, t) & =-e^{-\left\{\gamma x+\frac{1}{2} \frac{\mu^{2}}{\sigma^{2}}(T-t)\right\}} \\
\pi^{*} & =\frac{\mu}{\sigma \gamma} .
\end{aligned}
$$

For the seller's agent whose value function is Equation (9), the corresponding HJB equation is as follows

$$
\begin{array}{r}
V_{t}+\max _{\pi}\left(\mu \pi V_{x}+\frac{1}{2} \sigma^{2} \pi^{2} V_{x x}+\rho_{1} \sigma \pi v(R) V_{x r}+\rho_{2} \sigma \pi \vartheta(t) V_{x y}\right)+ \\
\varphi(R) V_{r}+\eta(Y, t) V_{y}++\rho v(R) \vartheta(t) V_{r y}+\frac{1}{2} v^{2}(R) V_{r r}+\frac{1}{2} \vartheta^{2}(t) V_{y y}=0 \\
V(T, x, r, y)=-e^{-\gamma[x-w(r, y)] .}
\end{array}
$$

Carrying out the optimization function by differentiating with respect to $\pi$ and since $\rho_{1}=\rho_{2}=0$ because the capital market is not correlated with the weather, we have the optimal control as

$$
\pi^{*}=-\frac{\mu}{\sigma^{2}} \frac{V_{x}}{V_{x x}}
$$

Substituting in the HJB equation [where we suppress the variables of function $g(y, t)=g$ ] yields

$$
\begin{array}{r}
V_{t}-\frac{1}{2} \frac{\mu^{2}}{\sigma^{2}} \frac{V_{x}^{2}}{V_{x x}}+\varphi V_{r}+\eta V_{y}+\rho v \vartheta(t) V_{r y}+\frac{1}{2} v^{2} V_{r r} \\
+\frac{1}{2} \vartheta^{2}(t) V_{y y}=0 \\
V(T, x, r, y)=-e^{-\gamma[x-w(r, y)] .}
\end{array}
$$

We postulate a solution of separable variables having the form $V(x, r, y, r, t)=-e^{-\gamma x} A(r, y, t)$ where the function $A(t, r, y)$ is twice differentiable in the second and third variables and square integrable. The HJB yields

$$
\begin{array}{r}
-e^{-\gamma x} A_{t}-\frac{1}{2} \frac{\mu^{2}}{\sigma^{2}} \frac{\left[\gamma e^{-\gamma x} A\right]^{2}}{\left[-\gamma^{2} e^{-\gamma x}\right] A}+\varphi\left[-e^{-\gamma x} A_{r}\right]+\eta\left[-e^{-\gamma x} A_{y}\right]+\rho v \vartheta(t)\left[-e^{-\gamma x} A_{r y}\right]+ \\
\frac{1}{2} v^{2}\left[-e^{-\gamma x} A_{r r}\right]+\frac{1}{2} \vartheta^{2}(t)\left[-e^{-\gamma x} A_{y y}\right]=0,
\end{array}
$$

which reduces to

$$
\begin{array}{r}
A_{t}-\frac{1}{2} \frac{\mu^{2}}{\sigma^{2}} A+\varphi A_{r}+\eta A_{y}+\rho v \vartheta(t) A_{r y}+\frac{1}{2} v^{2} A_{r r}+\frac{1}{2} \vartheta^{2}(t) A_{y y}=0 \\
A(r, y, T)=-e^{\gamma w(r, y)} .
\end{array}
$$

Applying the Feynman-Kac formula to the model in Equations (11) and (12), we find the value of $A(t, r, y)$ as

$$
A(t, r, y)=e^{-\frac{\mu^{2}}{2 \sigma^{2}}(T-t)} \mathbf{E}_{\mathbf{P}}\left[-e^{\gamma W_{T}\left(R_{T}, Y_{T}\right)} \mid R_{t}=r, Y_{t}=y\right],
$$


where $R$ and $Y$ are solutions, respectively, to

$$
\begin{aligned}
& d R_{s}=\varphi d s+v d W_{s}^{1} \\
& d Y_{s}=\eta d s+\vartheta d W_{s}^{2} .
\end{aligned}
$$

Proposition 3. The optimal value and control in the presence of weather hedging, for the seller's agent is, respectively,

$$
\begin{aligned}
V(t, x, r, y) & =-e^{-\gamma_{s} x-\frac{\mu^{2}}{2 \sigma^{2}}(T-t)} \mathbf{E}_{\mathbf{P}}\left[-e^{\gamma_{s} W_{T}\left(R_{T}, Y_{T}\right)} \mid X_{t}=x, R_{t}=r, Y_{t}=y\right] \\
\pi^{*} & =\frac{\mu}{\sigma \gamma_{s}}
\end{aligned}
$$

where the subscript s indicates that this is the risk aversion for the seller.

Proposition 4. The seller's utility indifference price is

$$
p^{s}(x, r, y, t)=\frac{1}{\gamma_{s}} \ln \left(\mathbf{E}_{\mathbf{P}}\left[e^{\gamma_{s} W_{T}\left(R_{T}, Y_{T}\right)} \mid X_{t}=x, R_{t}=r, Y_{t}=y\right]\right) .
$$

Proof. The utility indifference price for the seller is a function $p^{s}(x, r, y, t)$ that satisfies

$$
V(x, t)=V^{s}\left(x+p^{s}(x, r, y, t), r, y, t\right),
$$

i.e.,

$$
-e^{-\left\{\gamma_{s} x+\frac{1}{2} \frac{\mu^{2}}{\sigma^{2}}(T-t)\right\}}=-e^{-\gamma_{s}\left[x+p^{s}(x, r, y, t)\right]-\frac{\mu^{2}}{2 \sigma^{2}}(T-t)} \mathbf{E}_{\mathbf{P}}\left[-e^{\gamma_{s} W_{T}\left(R_{T}, Y_{T}\right)} \mid X_{t}=x, R_{t}=r, Y_{t}=y\right],
$$

which gives

$$
p^{s}(x, r, y, t)=\frac{1}{\gamma_{s}} \ln \left(\mathbf{E}_{\mathbf{P}}\left[e^{\gamma_{s} W_{T}\left(R_{T}, Y_{T}\right)} \mid X_{t}=x, R_{t}=r, Y_{t}=y\right]\right)
$$

Proposition 5. Following same reasoning, one can obtain the buyer's utility indifference price as

$$
p^{b}(x, r, y, t)=-\frac{1}{\gamma_{b}} \ln \left(\mathbf{E}_{\mathbf{P}}\left[e^{-\gamma_{b} W_{T}\left(R_{T}, Y_{T}\right)} \mid X_{t}=x, R_{t}=r, Y_{t}=y\right]\right) .
$$

The utility indifference prices $p^{s}(x, r, y, t)$ and $p^{b}(x, r, y, t)$ are clearly independent of initial wealth $x$ and is nonlinear, which agrees with Carmona (2008); Musiela and Zariphopoulou (2001). The nonlinearity of these prices implies that the buyer should not pay twice as much for twice as many options but requires a reduction in the price to take on the additional risks, whereas the seller requires more than twice the price for taking on twice the risk.

Another notable observation is that the presence of the traded asset is irrelevant from the perspective of risks to be hedged. This is because the correlation between the capital market and the weather indexes is negligible and hence the expectation is taken on physical probabilities $\mathbf{P}$ such that the indifference measure is the same as the historical measure. However, both prices are built on the investor's preference towards risks that cannot be be eliminated $\gamma$ due to market incompleteness. 
For the market to be viable and free of arbitrage opportunities, the market forward price $F(W)$ of the weather derivative must not be less than the seller's utility indifference price and similarly not more than the buyer's utility indifference price. Therefore, the utility indifference prices serve as the starting point for price negotiations for weather derivatives between potential buyers and sellers. They are a pricing (bid-ask spread) for a specific agent as currently weather derivatives are sold on the counter and not on the capital market.

\section{Hedging Maize Yield Using Basket Weather Derivative}

Maize is the most important grain crop in Malawi and accounts for almost $90 \%$ total calorie intake Dorward and Chirwa (2011). It is produced throughout the country under diverse environments. The maize crop requires around $450 \mathrm{~mm}$ of accumulated water per season, which runs for 140 days from November to mid-March mainly acquired from soil moisture Kawaye and Hutchinson (2018). It is a warm weather crop and does not grow well in areas where the mean daily temperature is less than $19^{\circ} \mathrm{C}$ or where the mean of the summer months is less than $23^{\circ} \mathrm{C}$.

High temperatures coupled with drought can significantly affect the pollination process, especially if it occurs during and within 10 days of pollination period. In addition, lack of rainfall or too much of it can reduce the maize yield by $100 \%$ Harrison et al. (2011), resulting in severe hunger and leaving a lot of people vulnerable and food insufficient.

In view of climate change, especially variability of rainfall and temperatures, there is a need to introduce weather derivatives as contingent measure to help farmers to hedge potential losses in maize yield. The study introduces basket weather derivatives whose underlying indexes are rainfall and temperature processes, which are arguably among the most important factors contributing to maize yield variability.

For the specification of the relationship between weather in terms of rainfall and temperature, and maize yield $Y$, the model by Vedenov et al. (2004) is:

$$
\begin{aligned}
Y & =I+\epsilon \quad \text { where } \\
I & =\alpha_{0}+\alpha_{2} R_{c d}+\alpha_{3} C D D+\alpha_{3} R_{c d}^{2}+\alpha_{4} C D D^{2}+\alpha_{5} R_{c d} C D D .
\end{aligned}
$$

Here, I denotes the weather index, and $\epsilon \sim N\left[0, \sigma^{2}\right]$.

$R_{c d}$ is cumulative rainfall deficit during the whole growing season defined as

$$
R_{c d}=\sum_{i=1}^{n} \min \left(0, \sum_{j=(i-1) s+1}^{i s} r_{i}-r^{\min }\right) .
$$

Equation (18) measures the shortfall of the sum of daily rainfall amounts $r_{i}$ during the period of $s$ days relative to the required amount $r^{\mathrm{min}}$. $C D D$ is the number of cumulative cooling degree days defined as the number of days in which the average daily temperature $T_{i}$ is more than the recommended temperature for maize growth at $23{ }^{\circ} \mathrm{C}$ given as

$$
C D D=\sum_{i=1}^{n} \mathbf{1}_{T_{i}>23}
$$

Estimation of parameters $\alpha_{0}, \ldots, \alpha_{5}$ can be based on the maize yield data while the weather variables should be derived from average daily temperature and daily rainfall data. 
Based on this information, a basket weather derivative can be designed that maximizes the hedging effectiveness of maize producers. Choosing a put option with pay-off $W_{T}$ at the expiration date $T$,

$$
W_{T}=\max \left(0, K-I_{T}\right) \times L,
$$

where $I_{T}$ is the weather index at expiration date, $K$ is the strike price of the option and $L$ is the tick size determined in such away that a negative correlation between pay-off of the option and the revenues of the maize production is maximal.

Utility indifference prices of the buyer and seller for the basket option were simulated where it was assumed that the option has a relative risk averse parameter of $\gamma=0.1$ since in most cases farmers are risk averse and would not be willing to take huge risks.

Rainfall and temperature data for 20 seasons were obtained from Balaka district in Malawi, for which we calculated utility indifference prices for the latest five years.

Table 1 shows the utility indifference prices for the seller and buyer of a basket weather derivative simulated using Equations (14) and (15) for rainy seasons from 2011 to 2016 . We assumed the same risk aversion for both seller and buyer.

Table 1. Utility indifference prices for basket weather derivatives.

\begin{tabular}{cccc}
\hline Year & Risk Averse & Seller's Price & Buyer's Price \\
\hline 2016 & 0.1 & 826.59 & 976.80 \\
2015 & 0.1 & 697.03 & 781.11 \\
2014 & 0.1 & 709.49 & 804.63 \\
2013 & 0.1 & 687.03 & 773.64 \\
2012 & 0.001 & 674.11 & 703.41 \\
2011 & 0.1 & 590.31 & 622.53 \\
\hline
\end{tabular}

The prices vary on each season depending on the rainfall and temperature of that particular year. The future price of a basket weather derivative then should be negotiated in between the two prices so that there is no arbitrage opportunities. The indifference prices for the seller constitute a lower bound for the fair asking price of the derivative. In reality there will be transaction costs for developing and launching the derivatives added to the prices. Furthermore the willingness for a farmer to buy the derivative may also depend on other factors like farm size and family income but the model has demonstrated that it is possible to hedge crop yield against risks due to rainfall and temperature in one transaction.

\section{Conclusions}

The weather derivative market is a classical incomplete market since the weather indexes are not tradable assets, thus traditional no arbitrage pricing methods such as the Black-Scholes are not applicable in pricing weather derivatives. In this paper, we adopt the utility indifference pricing approach where the investor's risk preference towards the risks that cannot be eliminated is described by a utility function, exponential in our case. The approach takes into account price risk, weather risks and all other risks in the financial capital market.

The buyer's and seller's indifference prices are derived and conditions for market viability and no arbitrage opportunities are derived, which all relate to the investor's risk aversion based on the utility function. In this paper, we assume that there is no correlation between the tradable asset and weather indexes, considering that we are interested in how a farmer can hedge weather risks related to rainfall and temperature simultaneously, and it is found that the indifference pricing measure is the same as the physical measure. 
The pricing model developed can be used in agriculture industry where a farmer is interested in hedging weather risks due to rainfall and temperature simultaneously and economically. It can also be used to price weather derivatives in other weather related industries affected by rainfall, temperature or both.

The model developed can be refined by ether choosing a different utility function such as power utility, which means a different risk preference by the investor, or taking into account the bond in the model $(r \neq 0)$. Since weather derivatives are traded on the counter, one may compare the prices developed here with those by actuarial approaches.

Author Contributions: The conceptualization of the research done by N.C.D. whereas the formal analysis was carried by P.N. with the supervision being done by L.O.

Funding: This research was funded by Pan African University.

Acknowledgments: The authors would like to thank Pan African University for the scholarship awarded to the first author.

Conflicts of Interest: The authors declare no conflict of interest.

\section{References}

Alexandridis, Antonis, and Achilleas D. Zapranis. 2012. Weather Derivatives: Modeling and Pricing Weather-Related Risk. Berlin: Springer Science \& Business Media.

Benth, Fred Espen, and Jūratė S̆altytè-Benth. 2005. Stochastic modelling of temperature variations with a view towards weather derivatives. Applied Mathematical Finance 12: 53-85. [CrossRef]

Benth, Fred Espen, and Jūratè šaltytė Benth. 2007. The volatility of temperature and pricing of weather derivatives. Quantitative Finance 7: 553-61. [CrossRef]

Benth, Fred Espen, and Jūratè Šaltytè Benth. 2011. Weather derivatives and stochastic modelling of temperature. International Journal of Stochastic Analysis 2011: 576791. [CrossRef]

Benth, Fred Espen, and Kenneth Hvistendahl Karlsen. 2005. A note on merton's portfolio selection problem for the schwartz mean-reversion model. Stochastic Analysis and Applications 23: 687-704. [CrossRef]

Bjork, Tomas. 2009. Arbitrage Theory in Continuous Time. OUP Catalogue. Oxford: Oxford University Press.

Brockett, Patrick L., Linda L. Goldens, Min-Ming Wen, and Charles C. Yang. 2009. Pricing weather derivatives using the indifference pricing approach. North American Actuarial Journal 13: 303-15. [CrossRef]

Brody, Dorje C., Joanna Syroka, and Mihail Zervos. 2002. Dynamical pricing of weather derivatives. Quantitative Finance 2: 189-98. [CrossRef]

Cao, Melanie, Anlong Li, and Jason Z. Wei. 2004. Precipitation modeling and contract valuation: A frontier in weather derivatives. The Journal of Alternative Investments 7: 93-99. [CrossRef]

Carmona, René. 2008. Indifference Pricing: Theory and Applications. Princeton: Princeton University Press.

Carmona, René, and Danilova Albina. 2003. Hedging financial instruments written on non-tradable indexes. Unpublished manuscript.

Carmona, Rene, and Pavel Diko. 2005. Pricing precipitation based derivatives. International Journal of Theoretical and Applied Finance 8: 959-88. [CrossRef]

Cong, Rong-Gang, and Mark Brady. 2012. The interdependence between rainfall and temperature: Copula analyses. The Scientific World Journal 2012: 405675. [CrossRef] [PubMed]

Cui, Kaijie. 2014. Weather Derivatives: Modelling, Pricing and Applications. Ph.D. Thesis, University of Calgary, Calgary, AB, Canada.

Dorward, Andrew, and Ephraim Chirwa. 2011. The malawi agricultural input subsidy programme: 2005/06 to 2008/09. International Journal of Agricultural Sustainability 9: 232-47. [CrossRef]

Eberlein, Ernst, and Jean Jacod. 1997. On the range of options prices. Finance and Stochastics 1: 131-40. [CrossRef]

Fleming, Wendell H., and Halil Mete Soner. 2006. Controlled Markov Processes and Viscosity Solutions. Berlin: Springer Science \& Business Media, vol. 25. 
Harrison, L., J. Michaelsen, Chris Funk, and G. Husak. 2011. Effects of temperature changes on maize production in mozambique. Climate Research 46: 211-22. [CrossRef]

Henderson, Vicky, and David Hobson. 2004. Utility indifference pricing-An overview. In Volume on Indifference Pricing. Princeton: Princeton University Press.

Hertzler, Greg. 2003. A stochastic differential equation for modeling the "classical" probability distributions. Paper presented at 2003 Conference (47th) Australian Agricultural and Resource Economics Society (AARES), Fremantle, Australia, February 12-14; pp. 12-14.

Kawaye, Floney P., and Michael F. Hutchinson. 2018. Are increases in maize production in malawi due to favourable climate or the farm input subsidy program? In Theory and Practice of Climate Adaptation. Cham: Springer, pp. 375-90.

López Cabrera, Brenda, Martin Odening, and Matthias Ritter. 2013. Pricing Rainfall Derivatives at the CME. Technical Report. Berlin: Humboldt University, Collaborative Research Center, p. 649.

Leobacher, Gunther, and Philip Ngare. 2011. On modelling and pricing rainfall derivatives with seasonality. Applied Mathematical Finance 18: 71-91. [CrossRef]

Medori, Mauro, Lucia Michelini, Isabel Nogues, Francesco Loreto, and Carlo Calfapietra. 2012. The impact of root temperature on photosynthesis and isoprene emission in three different plant species. The Scientific World Journal 2012: 525827. [CrossRef] [PubMed]

Musiela, Marek, and Thaleia Zariphopoulou. 2001. Indifference prices and related measures, preprint.

Musiela, Marek, and Thaleia Zariphopoulou. 2004. An example of indifference prices under exponential preferences. Finance and Stochastics 8: 229-39. [CrossRef]

Odening, Martin, Oliver Mußhoff, and Wei Xu. 2007. Analysis of rainfall derivatives using daily precipitation models: Opportunities and pitfalls. Agricultural Finance Review 67: 135-56. [CrossRef]

Ray, Deepak K., James S. Gerber, Graham K. MacDonald, and Paul C. West. 2015. Climate variation explains a third of global crop yield variability. Nature Communications 6: 5989. [CrossRef] [PubMed]

Syroka, Joanna, and Antonio Nucifora. 2010. National Drought Insurance for Malawi. Washington: The World Bank.

Turvey, Calum G. 2001. Weather derivatives for specific event risks in agriculture. In Review of Agricultural Economics. Oxford: Oxford University Press, pp. 333-51.

Vedenov, Dmitry V., and Barry J. Barnett. 2004. Efficiency of weather derivatives as primary crop insurance instruments. Journal of Agricultural and Resource Economics 29: 387-403.

Wang, Zhiliang, Peng Li, Lingyong Li, Chunyan Huang, and Min Liu. 2015. Modeling and forecasting average temperature for weather derivative pricing. Advances in Meteorology 2015: 837293. [CrossRef]

Xu, Wei, Martin Odening, and Oliver Mußhoff. 2007. Indifference pricing of weather insurance. Paper presented at 101st Seminar of the European Association of Agricultural Economists (EAAE) "Management of Climate Risks in Agriculture", Berlin, Germany, July 5-6.

Zscheischler, Jakob, Rene Orth, and Sonia I. Seneviratne. 2017. Bivariate return periods of temperature and precipitation explain a large fraction of european crop yields. Biogeosciences 14: 3309-20. [CrossRef]

(C) 2019 by the authors. Licensee MDPI, Basel, Switzerland. This article is an open access article distributed under the terms and conditions of the Creative Commons Attribution (CC BY) license (http:/ / creativecommons.org/licenses/by/4.0/). 